\title{
LMI Approach to Suboptimal Guaranteed Cost Control for 2-D Discrete Uncertain Systems
}

\author{
Amit Dhawan, Haranath Kar
}

Department of Electronics and Communication Engineering, Motilal Nehru National Institute of Technology, Allahabad, India. Email: \{amit_dhawan2, hnkar1\}@rediffmail.com

Received September $28^{\text {th }}, 2011$; revised October $30^{\text {th }}, 2011$; accepted November $14^{\text {th }}, 2011$.

\begin{abstract}
This paper studies the problem of the guaranteed cost control via static-state feedback controllers for a class of two-dimensional (2-D) discrete systems described by the Fornasini-Marchesini second local state-space (FMSLSS) model with norm bounded uncertainties. A convex optimization problem with linear matrix inequality (LMI) constraints is formulated to design the suboptimal guaranteed cost controller which ensures the quadratic stability of the closed-loop system and minimizes the associated closed-loop cost function. Application of the proposed controller design method is illustrated with the help of one example.
\end{abstract}

Keywords: Linear Matrix Inequality, Lyapunov Methods, Robust Stability, 2-D Discrete Systems, Uncertain Systems, Fornasini-Marchesini Second Local State-Space Model

\section{Introduction}

In the past few years, due to the rapid increase of a wide variety of applications of two-dimensional (2-D) discrete systems in many practical application domains such as digital filtering, image and video processing, seismographic data processing, thermal processes, gas absorption, water stream heating, control systems etc. [1-10], there has emerged a continuously growing interest in the system theoretic problems of 2-D discrete systems. Many authors have proposed and analyzed linear state-variable models for 2-D discrete systems [11-14]. The more popular models are Roesser model [11], Fornasini-Marchesini first model [13] and Fornasini-Marchesini second local state-space (FMSLSS) model [14]. Many publications relating to 2-D Lyapunov equation with constant coefficients for the Roesser model [11] have appeared [15-22]. The stability properties of 2-D discrete systems described by the FM first model [13] have been investigated extensively [23-29]. The stability analysis of 2-D discrete systems described by the FMSLSS model [14] has attracted a great deal of interest and many significant results have been obtained [22,30-44].

Due to assumptions in the modeling process and/or the changing operating conditions of a real world system, it is usually impossible for a mathematical model to describe the real world system exactly. The problem of designing robust controllers for 2-D uncertain systems has drawn the attention of several researchers in recent years $[39,40]$. When controlling a system subject to parameter uncertainty, it is also desirable to design a control system which is not only stable but also guarantees an adequate level of performance. One approach to this problem is the socalled guaranteed cost control approach [45]. This approach has the advantage of providing an upper bound on a given performance index and thus the system performance degradation incurred by the uncertainties is guaranteed to be less than this bound. Based on this idea, many significant results have been proposed [42-51]. In [42-44], the guaranteed cost control problem for 2-D discrete uncertain systems in FMSLSS setting has been considered and a robust controller design method has been established. The approach of [42] does not provide a true linear matrix inequality (LMI) based result which is not beneficial in terms of numerical complexity. Subsequently, in [43], an LMI based criterion for the existence of robust guaranteed cost controller has been formulated. Robust suboptimal guaranteed cost control for 2-D discrete uncertain systems in FMSLSS setting is an important problem.

In recent years, LMI has emerged as a powerful tool in control design problems [52-58]. The introduction of LMI in control theory has given a new direction in the area of robust control problems. A widely accepted method for solving robust control problems now is to simply 
reduce them to LMI problems. Since solving LMIs is a convex optimization problem, such formulations offer a numerically efficient means of attacking problems that are difficult to solve analytically. These LMIs can be solved effectively by employing the recently developed Matlab LMI toolbox [53].

This paper, therefore, deals with the suboptimal guaranteed cost control problem for 2-D discrete uncertain systems described by FMSLSS model with norm-bounded uncertainties. The paper is organized as follows. In Section 2 , we formulate the problem of robust guaranteed cost control for the uncertain 2-D discrete system described by the FMSLSS model and recall some useful results. An LMI based approach for the design of suboptimal guaranteed cost controller via static-state feedback is presented in Section 3. In Section 4, an application of the presented robust guaranteed cost controller design method is given. Finally, some concluding remarks are given in Section 5.

\section{Problem Formulation and Preliminaries}

The following notations are used throughout the paper:

$$
\begin{aligned}
& \boldsymbol{R}^{\boldsymbol{n}} \quad \text { real vector space of dimension } n \\
& \boldsymbol{R}^{n \times m} \quad \text { set of } n \times m \text { real matrices } \\
& 0 \text { null matrix or null vector of } \\
& \text { appropriate dimension } \\
& \text { I identity matrix of appropriate } \\
& \boldsymbol{G}^{\boldsymbol{T}} \quad \text { transpose of matrix } \boldsymbol{G} \\
& \boldsymbol{G}>\mathbf{0} \quad \text { matrix } \boldsymbol{G} \text { is positive definite symmetric } \\
& \boldsymbol{G}<\mathbf{0} \text { matrix } \boldsymbol{G} \text { is negative definite symmetric } \\
& \operatorname{det}(\boldsymbol{G}) \quad \text { determinant of matrix } \boldsymbol{G} \\
& \lambda_{\text {max }}(\boldsymbol{G}) \quad \text { maximum eigenvalue of matrix } \boldsymbol{G} \text {. } \\
& \boldsymbol{x}(i+1, j+1)=\left(A_{1}+\Delta \boldsymbol{A}_{1}\right) \boldsymbol{x}(i+1, j) \\
& +\left(A_{2}+\Delta A_{2}\right) \boldsymbol{x}(i, j+1) \\
& +\left(\boldsymbol{B}_{1}+\Delta \boldsymbol{B}_{1}\right) \boldsymbol{u}(i+1, j), \\
& +\left(\boldsymbol{B}_{2}+\Delta \boldsymbol{B}_{2}\right) \boldsymbol{u}(i, j+1) \\
& \boldsymbol{A}=\left[\begin{array}{ll}
\boldsymbol{A}_{1} & \boldsymbol{A}_{2}
\end{array}\right],
\end{aligned}
$$

where $\boldsymbol{x}(i, j) \in R^{n}$ and $\boldsymbol{u}(i, j) \in R^{m}$ are the state and control input, respectively. The matrices $\boldsymbol{A}_{k} \in R^{n \times n}$ and $\boldsymbol{B}_{k} \in R^{n \times m} \quad(k=1,2)$ are known constant matrices representing the nominal plant, $\Delta \boldsymbol{A}_{k}$ and $\Delta \boldsymbol{B}_{k}(k=1,2)$ are real valued matrix functions representing parameter uncertainties in the system model. The parameter uncertainties under consideration are assumed to be norm-bounded and of the form

$$
\left[\begin{array}{ll}
\Delta \boldsymbol{A} & \Delta \boldsymbol{B}
\end{array}\right]=\boldsymbol{L} \boldsymbol{F}(i, j)\left[\begin{array}{ll}
\boldsymbol{M}_{1} & \boldsymbol{M}_{2}
\end{array}\right],
$$

where

$$
\begin{array}{ll}
\Delta \boldsymbol{A}=\left[\begin{array}{ll}
\Delta \boldsymbol{A}_{1} & \Delta \boldsymbol{A}_{2}
\end{array}\right] & \Delta \boldsymbol{B}=\left[\begin{array}{ll}
\Delta \boldsymbol{B}_{1} & \Delta \boldsymbol{B}_{2}
\end{array}\right], \\
\boldsymbol{M}_{1}=\left[\begin{array}{ll}
\boldsymbol{M}_{11} & \boldsymbol{M}_{12}
\end{array}\right] & \boldsymbol{M}_{2}=\left[\begin{array}{ll}
\boldsymbol{M}_{21} & \boldsymbol{M}_{22}
\end{array}\right] .
\end{array}
$$

In the above, $\boldsymbol{L}, \boldsymbol{M}_{1}$ and $\boldsymbol{M}_{2}$ can be regarded as known structural matrices of uncertainty and $\boldsymbol{F}(i, j)$ is an unknown matrix representing parameter uncertainty which satisfies

$$
\|\boldsymbol{F}(i, j)\| \leq 1 .
$$

It may be mentioned that the uncertainty of (1c) satisfying (1f) has been widely adopted in robust control literature [38,39,42-44,59-62]. The matrices $\boldsymbol{L}$ and $\boldsymbol{M}_{1}$ $\left(\boldsymbol{M}_{2}\right)$ specify how the elements of the nominal matrices $\boldsymbol{A}(\boldsymbol{B})$ are affected by the uncertain parameters in $\boldsymbol{F}(i, j)$. Note that $\boldsymbol{F}(i, j)$ can always be restricted as (1f) by appropriately selecting $\boldsymbol{L}, \boldsymbol{M}_{1}$ and $\boldsymbol{M}_{2}$. Therefore, there is no loss of generality in choosing $\boldsymbol{F}(i, j)$ as in (1f).

It is assumed that the system (1a) has a finite set of initial conditions $[22,34,36,38,43,44]$ i.e., there exist two positive integers $p$ and $q$ such that

$$
\boldsymbol{x}(i, 0)=\mathbf{0}, \quad i \geq p ; \quad \boldsymbol{x}(0, j)=\mathbf{0}, \quad j \geq q,
$$

and the initial conditions are arbitrary, but belong to the set [42-44]

$$
\begin{gathered}
S=\left\{\boldsymbol{x}(i, 0), \boldsymbol{x}(0, j) \in R^{n}: \boldsymbol{x}(i, 0)=\boldsymbol{M} \boldsymbol{N}_{1},\right. \\
\left.\boldsymbol{x}(0, j)=\boldsymbol{M} \boldsymbol{N}_{2}, \quad \boldsymbol{N}_{k}^{T} \boldsymbol{N}_{k}<1 \quad(k=1,2)\right\},
\end{gathered}
$$

where $\boldsymbol{M}$ is a given matrix.

Associated with the uncertain system (1) is the cost function $[43,44]$ :

$$
\begin{aligned}
J & =\sum_{i=0}^{\infty} \sum_{j=0}^{\infty}\left[\boldsymbol{u}^{T}(i+1, j) \boldsymbol{R}_{1} \boldsymbol{u}(i+1, j)\right. \\
& \left.+\boldsymbol{u}^{T}(i, j+1) \boldsymbol{R}_{2} \boldsymbol{u}(i, j+1)\right], \\
& +\sum_{i=0}^{\infty} \sum_{j=0}^{\infty} \xi_{i j}{ }^{T} \boldsymbol{W}_{1} \xi_{i j}
\end{aligned}
$$

where

$$
\begin{gathered}
\xi_{i j}=\left[\begin{array}{l}
\boldsymbol{x}(i+1, j) \\
\boldsymbol{x}(i, j+1)
\end{array}\right], \\
\mathbf{0}<\boldsymbol{R}_{k}=\boldsymbol{R}_{k}{ }^{T} \in R^{m \times m} \quad(k=1,2), \\
\boldsymbol{W}_{1}=\left[\begin{array}{cc}
\boldsymbol{Q}_{1} & \mathbf{0} \\
\mathbf{0} & \boldsymbol{Q}_{2}
\end{array}\right], \\
\mathbf{0}<\boldsymbol{Q}_{k}=\boldsymbol{Q}_{k}{ }^{T} \in R^{n \times n} \quad(k=1,2) .
\end{gathered}
$$

Suppose the system state is available for feedback, the objective of this paper is to develop a procedure to de- 
sign a static-state feedback control law

$$
\boldsymbol{u}(i, j)=\boldsymbol{K} \boldsymbol{x}(i, j)
$$

for the system (1) and the cost function (2), such that the closed-loop system

$$
\begin{aligned}
\boldsymbol{x}(i+1, j+1) & =\left[\left(\boldsymbol{A}_{1}+\boldsymbol{B}_{1} \boldsymbol{K}\right)+\left(\Delta \boldsymbol{A}_{1}+\Delta \boldsymbol{B}_{1} \boldsymbol{K}\right)\right] \boldsymbol{x}(i+1, j) \\
& +\left[\left(\boldsymbol{A}_{2}+\boldsymbol{B}_{2} \boldsymbol{K}\right)+\left(\Delta \boldsymbol{A}_{2}+\Delta \boldsymbol{B}_{2} \boldsymbol{K}\right)\right] \boldsymbol{x}(i, j+1)
\end{aligned}
$$

is asymptotically stable and the closed-loop cost function $J$ is minimized where

$$
\begin{gathered}
J=\sum_{i=0}^{\infty} \sum_{j=0}^{\infty} \xi_{i j}{ }^{T} \boldsymbol{W}_{2} \xi_{i j}, \\
\boldsymbol{W}_{2}=\left[\begin{array}{cc}
\boldsymbol{Q}_{1}+\boldsymbol{K}^{T} \boldsymbol{R}_{1} \boldsymbol{K} & \mathbf{0} \\
\mathbf{0} & \boldsymbol{Q}_{2}+\boldsymbol{K}^{T} \boldsymbol{R}_{2} \boldsymbol{K}
\end{array}\right] .
\end{gathered}
$$

Definition 2.1 A control law (3) is said to be an optimal quadratic guaranteed cost control if it ensures the quadratic stability of the closed-loop system (4) and minimizes the closed-loop cost function (5).

As an extension of the result for the global asymptotic stability condition of 2-D discrete FMSLSS model given in [14,30-33], one can easily arrive at the following lemma.

Lemma 2.1 [44] The 2-D discrete uncertain system (1) is globally asymptotically stable if and only if

$$
\operatorname{det}\left\{\boldsymbol{I}-z_{2}\left(\boldsymbol{A}_{1}+\boldsymbol{L} \boldsymbol{F} \boldsymbol{M}_{11}\right)-z_{1}\left(\boldsymbol{A}_{2}+\boldsymbol{L} \boldsymbol{F} \boldsymbol{M}_{12}\right)\right\} \neq 0
$$

for all

$$
\left(z_{1}, z_{2}, \boldsymbol{F}\right) \in \overline{\mathrm{U}^{2}},
$$

where $\overline{\mathrm{U}^{2}}=\left\{\left(z_{1}, z_{2}, \boldsymbol{F}\right):\left|z_{1}\right| \leq 1,\left|z_{2}\right| \leq 1,\|\boldsymbol{F}\| \leq 1\right\}$.

Definition 2.2 [42-44] Consider the uncertain system (1) and cost function (2), then the static-state feedback controller $\boldsymbol{u}(i, j)=\boldsymbol{K} \boldsymbol{x}(i, j)$ is said to define a quadratic guaranteed cost control associated with cost matrix $\mathbf{0}<\boldsymbol{P}=\boldsymbol{P}^{T} \in R^{n \times n}$ if there exist a $2 n \times 2 n$ positive definite symmetric matrix $\boldsymbol{W}_{2}$ given by $(5 \mathrm{~b})$ and an $n \times n$ positive definite symmetric matrix $\boldsymbol{P}_{1}$ such that

$$
\boldsymbol{\Gamma}_{C L}+\boldsymbol{W}_{2}<\mathbf{0},
$$

where

$$
\begin{aligned}
\boldsymbol{\Gamma}_{C L} & =\left[\begin{array}{ll}
\boldsymbol{A}_{\Delta 1}+\boldsymbol{B}_{\Delta 1} \boldsymbol{K} & \boldsymbol{A}_{\Delta 2}+\boldsymbol{B}_{\Delta 2} \boldsymbol{K}
\end{array}\right]^{T} \boldsymbol{P} \\
& \times\left[\begin{array}{ll}
\boldsymbol{A}_{\Delta 1}+\boldsymbol{B}_{\Delta 1} \boldsymbol{K} & \boldsymbol{A}_{\Delta 2}+\boldsymbol{B}_{\Delta 2} \boldsymbol{K}
\end{array}\right]-\left[\begin{array}{cc}
\boldsymbol{P}_{1} & \mathbf{0} \\
\mathbf{0} & \boldsymbol{P}-\boldsymbol{P}_{1}
\end{array}\right]
\end{aligned}
$$

and

$$
\begin{gathered}
\boldsymbol{A}_{\Delta 1}=\boldsymbol{A}_{1}+\Delta \boldsymbol{A}_{1}=\boldsymbol{A}_{1}+\boldsymbol{L} \boldsymbol{F} \boldsymbol{M}_{11}, \\
\boldsymbol{A}_{\Delta 2}=\boldsymbol{A}_{2}+\Delta \boldsymbol{A}_{2}=\boldsymbol{A}_{2}+\boldsymbol{L} \boldsymbol{F} \boldsymbol{M}_{12}, \\
\boldsymbol{B}_{\Delta 1}=\boldsymbol{B}_{1}+\Delta \boldsymbol{B}_{1}=\boldsymbol{B}_{1}+\boldsymbol{L} \boldsymbol{F} \boldsymbol{M}_{21},
\end{gathered}
$$

$$
\boldsymbol{B}_{\Delta 2}=\boldsymbol{B}_{2}+\Delta \boldsymbol{B}_{2}=\boldsymbol{B}_{2}+\boldsymbol{L} \boldsymbol{F} \boldsymbol{M}_{22} .
$$

The following lemmas are needed in the proof of our main result.

Lemma $2.2[42,44,51]$ Let $\boldsymbol{A} \in R^{n \times n}, \boldsymbol{H} \in R^{n \times k}$, $\boldsymbol{E} \in R^{l \times n}$ and $\boldsymbol{Q}=\boldsymbol{Q}^{T} \in R^{n \times n}$ be given matrices. Then there exists a positive definite matrix $\boldsymbol{P}$ such that

$$
[\boldsymbol{A}+\boldsymbol{H} \boldsymbol{F} \boldsymbol{E}]^{T} \boldsymbol{P}[\boldsymbol{A}+\boldsymbol{H} \boldsymbol{F} \boldsymbol{E}]-\boldsymbol{Q}<\mathbf{0}
$$

for all $\boldsymbol{F}$ satisfying $\boldsymbol{F}^{T} \boldsymbol{F} \leq \boldsymbol{I}$, if and only if there exists a scalar $\varepsilon>0$ such that

$$
\left[\begin{array}{cc}
-\boldsymbol{P}^{-1}+\varepsilon \boldsymbol{H} \boldsymbol{H}^{T} & \boldsymbol{A} \\
\boldsymbol{A}^{T} & \varepsilon^{-1} \boldsymbol{E}^{T} \boldsymbol{E}-\boldsymbol{Q}
\end{array}\right]<\mathbf{0} .
$$

Lemma $2.3[52,63]$ For real matrices $\boldsymbol{M}, \boldsymbol{L}, \boldsymbol{Q}$ of appropriate dimensions, where $\boldsymbol{M}=\boldsymbol{M}^{T}$ and $\boldsymbol{Q}=\boldsymbol{Q}^{T}>\mathbf{0}$, then $\boldsymbol{M}+\boldsymbol{L}^{T} \boldsymbol{Q} \boldsymbol{L}<\mathbf{0}$ if and only if

$$
\left[\begin{array}{cc}
\boldsymbol{M} & \boldsymbol{L}^{T} \\
\boldsymbol{L} & -\boldsymbol{Q}^{-1}
\end{array}\right]<\mathbf{0}
$$

or equivalently

$$
\left[\begin{array}{cc}
-\boldsymbol{Q}^{-1} & \boldsymbol{L} \\
\boldsymbol{L}^{T} & \boldsymbol{M}
\end{array}\right]<\mathbf{0} .
$$

Lemma 2.4 [44] Suppose there exists a quadratic guaranteed cost matrix $\mathbf{0}<\boldsymbol{P}=\boldsymbol{P}^{T}$ for the uncertain closedloop system (4) with initial conditions (1g), (1h) and cost function (5) such that (7) holds. Then, a) the uncertain closed-loop system (4) is quadratically stable and b) the cost function satisfies the bound

$$
J \leq(p+q-2) \lambda_{\max }\left(\boldsymbol{M}^{T} \boldsymbol{P} \boldsymbol{M}\right)
$$

\section{Main Result}

In this section, we establish that the problem of determining quadratic guaranteed cost control for system (1) and cost function (2) can be recast to a convex optimization problem. The main result may be stated as follows.

Theorem 3.1 Consider system (1) and cost function (2), then there exists a suboptimal static-state feedback controller $\boldsymbol{u}(i, j)=\boldsymbol{K} \boldsymbol{x}(i, j)$ that solves the addressed robust guaranteed cost control problem if the following optimization problem

minimize $(\varepsilon+\lambda)$

$$
\text { s.t. }\left\{\begin{array}{l}
(\text { i }) .(13), \\
\text { (ii). }\left[\begin{array}{cc}
-\lambda \boldsymbol{I} & \boldsymbol{M}^{T} \\
\boldsymbol{M} & -\boldsymbol{S}
\end{array}\right]<\mathbf{0}
\end{array}\right.
$$

has a feasible solution $\varepsilon>0, \boldsymbol{U} \in R^{m \times n}$, $\mathbf{0}<\boldsymbol{S}=\boldsymbol{S}^{\boldsymbol{T}} \in R^{n \times n}$ and $\mathbf{0}<\boldsymbol{Y}=\boldsymbol{Y}^{\boldsymbol{T}} \in R^{n \times n}$. The constraint (13) is given by 


$$
\left[\begin{array}{ccccccccc}
-\boldsymbol{S} & \overline{\boldsymbol{A}}_{1} & \overline{\boldsymbol{A}}_{2} & \boldsymbol{L} & \mathbf{0} & \mathbf{0} & \mathbf{0} & \mathbf{0} & \mathbf{0} \\
\overline{\boldsymbol{A}}_{1}^{T} & -\boldsymbol{Y} & \mathbf{0} & \mathbf{0} & \overline{\boldsymbol{M}}_{11} & \boldsymbol{S} \boldsymbol{Q}_{1}^{1 / 2} & \mathbf{0} & \boldsymbol{U}^{T} \boldsymbol{R}_{1}^{1 / 2} & \mathbf{0} \\
\overline{\boldsymbol{A}}_{2}^{T} & \mathbf{0} & -(\boldsymbol{S}-\boldsymbol{Y}) & \mathbf{0} & \overline{\boldsymbol{M}}_{12} & \mathbf{0} & \boldsymbol{S} \boldsymbol{Q}_{2}^{1 / 2} & \mathbf{0} & \boldsymbol{U}^{T} \boldsymbol{R}_{2}^{1 / 2} \\
\boldsymbol{L}^{T} & \mathbf{0} & \mathbf{0} & -\boldsymbol{I} & \mathbf{0} & \mathbf{0} & \mathbf{0} & \mathbf{0} & \mathbf{0} \\
\mathbf{0} & \overline{\boldsymbol{M}}_{11}^{T} & \overline{\boldsymbol{M}}_{12}^{T} & \mathbf{0} & -\boldsymbol{I} & \mathbf{0} & \mathbf{0} & \mathbf{0} & \mathbf{0} \\
\mathbf{0} & \boldsymbol{Q}_{1}^{T / 2} \boldsymbol{S} & \mathbf{0} & \mathbf{0} & \mathbf{0} & -\varepsilon \boldsymbol{I} & \mathbf{0} & \mathbf{0} & \mathbf{0} \\
\mathbf{0} & 0 & \boldsymbol{Q}_{2}^{T / 2} \boldsymbol{S} & \mathbf{0} & \mathbf{0} & \mathbf{0} & -\varepsilon \boldsymbol{I} & \mathbf{0} & \mathbf{0} \\
\mathbf{0} & \boldsymbol{R}_{1}^{T / 2} \boldsymbol{U} & \mathbf{0} & \mathbf{0} & \mathbf{0} & \mathbf{0} & \mathbf{0} & -\varepsilon \boldsymbol{I} & \mathbf{0} \\
\mathbf{0} & \mathbf{0} & \boldsymbol{R}_{2}^{T / 2} \boldsymbol{U} & \mathbf{0} & \mathbf{0} & \mathbf{0} & \mathbf{0} & \mathbf{0} & -\varepsilon \boldsymbol{I}
\end{array}\right]<\mathbf{0}
$$

where

$$
\begin{array}{r}
\overline{\boldsymbol{A}}_{1}=\boldsymbol{A}_{1} \boldsymbol{S}+\boldsymbol{B}_{1} \boldsymbol{U}, \\
\overline{\boldsymbol{A}}_{2}=\boldsymbol{A}_{2} \boldsymbol{S}+\boldsymbol{B}_{2} \boldsymbol{U}, \\
\overline{\boldsymbol{M}}_{11}=\boldsymbol{S} \boldsymbol{M}_{11}^{T}+\boldsymbol{U}^{T} \boldsymbol{M}_{21}^{T}, \\
\overline{\boldsymbol{M}}_{12}=\boldsymbol{S} \boldsymbol{M}_{12}^{T}+\boldsymbol{U}^{T} \boldsymbol{M}_{22}^{T} .
\end{array}
$$

In this situation, a suboptimal control law is $\boldsymbol{K}=\boldsymbol{U} \boldsymbol{S}^{-1}$ which ensures the minimization of the upper bound of (2) for the closed-loop uncertain system.

Proof: Using (5b) and (7b), matrix Inequality (7a) can be expressed as

$$
\begin{aligned}
& {\left[\begin{array}{ll}
\boldsymbol{A}_{\Delta 1}+\boldsymbol{B}_{\Delta 1} \boldsymbol{K} & \boldsymbol{A}_{\Delta 2}+\boldsymbol{B}_{\Delta 2} \boldsymbol{K}
\end{array}\right]^{T} \boldsymbol{P}} \\
& \times\left[\begin{array}{cc}
\boldsymbol{A}_{\Delta 1}+\boldsymbol{B}_{\Delta 1} \boldsymbol{K} & \boldsymbol{A}_{\Delta 2}+\boldsymbol{B}_{\Delta 2} \boldsymbol{K}
\end{array}\right]-\left[\begin{array}{cc}
\boldsymbol{P}_{1} & \mathbf{0} \\
\mathbf{0} & \boldsymbol{P}-\boldsymbol{P}_{1}
\end{array}\right], \\
& +\left[\begin{array}{cc}
\boldsymbol{Q}_{1}+\boldsymbol{K}^{T} \mathbf{R}_{1} \boldsymbol{K} & \mathbf{0} \\
\mathbf{0} & \boldsymbol{Q}_{2}+\boldsymbol{K}^{T} \mathbf{R}_{2} \boldsymbol{K}
\end{array}\right]<\mathbf{0}
\end{aligned}
$$

which, in view of (7c)-(7f), takes the form

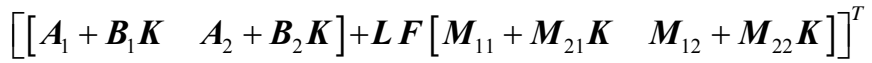

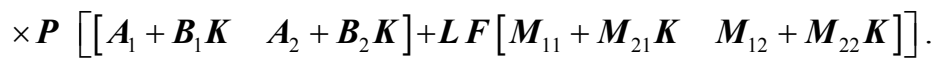

$$
\begin{aligned}
& +\left[\begin{array}{cc}
-\boldsymbol{P}_{1}+\boldsymbol{Q}_{1}+\boldsymbol{K}^{T} \mathbf{R}_{1} \boldsymbol{K} & \mathbf{0} \\
\mathbf{0} & -\left(\boldsymbol{P}-\boldsymbol{P}_{1}\right)+\boldsymbol{Q}_{2}+\boldsymbol{K}^{T} \mathbf{R}_{2} \boldsymbol{K}
\end{array}\right]<\mathbf{0}
\end{aligned}
$$

Applying Lemma 2.2, (17) can be rearranged as

$$
\begin{array}{cc}
{\left[\begin{array}{cc}
-\boldsymbol{P}^{-1}+\varepsilon^{-1} \mathbf{L} \mathbf{L}^{T} & \left(\boldsymbol{A}_{1}+\boldsymbol{B}_{1} \boldsymbol{K}\right) \\
\left(\boldsymbol{A}_{1}+\boldsymbol{B}_{1} \boldsymbol{K}\right)^{T} & -\boldsymbol{P}_{1}+\mathbf{Q}_{1}+\boldsymbol{K}^{T} \boldsymbol{R}_{1} \boldsymbol{K}+\varepsilon\left(\boldsymbol{M}_{11}+\boldsymbol{M}_{21} \boldsymbol{K}\right)^{T}\left(\boldsymbol{M}_{11}+\boldsymbol{M}_{21} \boldsymbol{K}\right) \\
\left(\boldsymbol{A}_{2}+\boldsymbol{B}_{2} \boldsymbol{K}\right)^{T} & \varepsilon\left(\boldsymbol{M}_{12}+\boldsymbol{M}_{22} \boldsymbol{K}\right)^{T}\left(\boldsymbol{M}_{11}+\boldsymbol{M}_{21} \boldsymbol{K}\right) \\
\varepsilon\left(\boldsymbol{M}_{11}+\boldsymbol{M}_{21} \boldsymbol{K}\right)^{T}\left(\boldsymbol{M}_{12}+\boldsymbol{M}_{22} \boldsymbol{K}\right) \\
-\left(\boldsymbol{P}-\boldsymbol{P}_{1}\right)+\mathbf{Q}_{2}+\boldsymbol{K}^{T} \boldsymbol{R}_{2} \boldsymbol{K}+\varepsilon\left(\boldsymbol{M}_{12}+\boldsymbol{M}_{22} \boldsymbol{K}\right)^{T}\left(\boldsymbol{M}_{12}+\boldsymbol{M}_{22} \boldsymbol{K}\right)
\end{array}\right]<\mathbf{0 .}}
\end{array}
$$

Premultiplying and postmultiplying (18) by the matrix $\left[\begin{array}{ccc}\varepsilon^{1 / 2} \boldsymbol{I} & \mathbf{0} & \mathbf{0} \\ \mathbf{0} & \varepsilon^{1 / 2} \boldsymbol{P}^{-1} & \mathbf{0} \\ \mathbf{0} & \mathbf{0} & \varepsilon^{1 / 2} \boldsymbol{P}^{-1}\end{array}\right]$, one obtains

$$
\left.\begin{array}{c}
{\left[\begin{array}{cc}
-\varepsilon \boldsymbol{P}^{-1}+\boldsymbol{L} \boldsymbol{L}^{T} & \varepsilon\left(\boldsymbol{A}_{1}+\boldsymbol{B}_{1} \boldsymbol{K}\right) \boldsymbol{P}^{-1} \\
\varepsilon \boldsymbol{P}^{-1}\left(\boldsymbol{A}_{1}+\boldsymbol{B}_{1} \boldsymbol{K}\right)^{T} & \varepsilon \boldsymbol{P}^{-1}\left\{-\boldsymbol{P}_{1}+\boldsymbol{Q}_{1}+\boldsymbol{K}^{T} \boldsymbol{R}_{1} \boldsymbol{K}+\varepsilon\left(\boldsymbol{M}_{11}+\boldsymbol{M}_{21} \boldsymbol{K}\right)^{T}\left(\boldsymbol{M}_{11}+\boldsymbol{M}_{21} \boldsymbol{K}\right)\right\} \boldsymbol{P}^{-1} \\
\varepsilon \boldsymbol{P}^{-1}\left(\boldsymbol{A}_{2}+\boldsymbol{B}_{2} \boldsymbol{K}\right)^{T} & \varepsilon \boldsymbol{P}^{-1}\left(\boldsymbol{M}_{12}+\boldsymbol{M}_{22} \boldsymbol{K}\right)^{T}\left(\boldsymbol{M}_{11}+\boldsymbol{M}_{21} \boldsymbol{K}\right) \varepsilon \boldsymbol{P}^{-1} \\
\varepsilon\left(\boldsymbol{A}_{2}+\boldsymbol{B}_{2} \boldsymbol{K}\right) \boldsymbol{P}^{-1} \\
\varepsilon \boldsymbol{P}^{-1}\left(\boldsymbol{M}_{11}+\boldsymbol{M}_{21} \boldsymbol{K}\right)^{T}\left(\boldsymbol{M}_{12}+\boldsymbol{M}_{22} \boldsymbol{K}\right) \varepsilon \boldsymbol{P}^{-1}
\end{array}\right]<\mathbf{0}} \\
\varepsilon \boldsymbol{P}^{-1}\left\{-\left(\boldsymbol{P}-\boldsymbol{P}_{1}\right)+\boldsymbol{Q}_{2}+\boldsymbol{K}^{T} \boldsymbol{R}_{2} \boldsymbol{K}+\varepsilon\left(\boldsymbol{M}_{12}+\boldsymbol{M}_{22} \boldsymbol{K}\right)^{T}\left(\boldsymbol{M}_{12}+\boldsymbol{M}_{22} \boldsymbol{K}\right)\right\} \boldsymbol{P}^{-1}
\end{array}\right]
$$


which can be rewritten as

$$
\begin{aligned}
& {\left[\begin{array}{cc}
-\boldsymbol{S}+\boldsymbol{L} \boldsymbol{L}^{T} & \overline{\boldsymbol{A}}_{1} \\
\overline{\boldsymbol{A}}_{1}^{T} & -\boldsymbol{Y}+\boldsymbol{\varepsilon}^{-1} \boldsymbol{S} \boldsymbol{Q}_{1} \boldsymbol{S}+\varepsilon^{-1} \boldsymbol{U}^{T} \boldsymbol{R}_{1} \boldsymbol{U}+\overline{\boldsymbol{M}}_{11} \overline{\boldsymbol{M}}_{11}^{T} \\
\overline{\boldsymbol{A}}_{2}^{T} & \overline{\boldsymbol{M}}_{12} \overline{\boldsymbol{M}}_{11}^{T}
\end{array}\right.} \\
& \left.\begin{array}{c}
\overline{\boldsymbol{A}}_{2} \\
\overline{\boldsymbol{M}}_{11} \overline{\boldsymbol{M}}_{12}^{T} \\
-(\boldsymbol{S}-\boldsymbol{Y})+\boldsymbol{\varepsilon}^{-1} \boldsymbol{S} \boldsymbol{Q}_{2} \boldsymbol{S}+\varepsilon^{-1} \boldsymbol{U}^{T} \boldsymbol{R}_{2} \boldsymbol{U}+\overline{\boldsymbol{M}}_{12} \overline{\boldsymbol{M}}_{12}^{T}
\end{array}\right]<\mathbf{0}
\end{aligned}
$$

where

$$
\begin{array}{r}
\boldsymbol{S}=\varepsilon \boldsymbol{P}^{-1}, \\
\boldsymbol{Y}=\varepsilon^{-1} \boldsymbol{S} \boldsymbol{P}_{1} \boldsymbol{S},
\end{array}
$$

and $\overline{\boldsymbol{A}}_{1}, \overline{\boldsymbol{A}}_{2}, \overline{\boldsymbol{M}}_{11}$ and $\overline{\boldsymbol{M}}_{12}$ are defined in (15).

Equation (19) can be expressed as Equation (22).

The equivalence of (22) and (13) follows trivially from Lemma 2.3. Using (20), the bound of the cost function (12) becomes

$$
J \leq(p+q-2) \varepsilon \lambda_{\max }\left(\boldsymbol{M}^{T} \boldsymbol{S}^{-1} \boldsymbol{M}\right) .
$$

Clearly, the upper bound (23) is not a convex function in $\boldsymbol{S}^{-1}$ and $\varepsilon$. Hence, finding the minimum of this upper bound can not be considered as a convex optimization problem. Since $\varepsilon$ and $\lambda_{\max }\left(\boldsymbol{M}^{T} \boldsymbol{S}^{-1} \boldsymbol{M}\right)$ are positive, we may obtain a suboptimal guaranteed cost controller by minimizing $\varepsilon+\lambda_{\max }\left(\boldsymbol{M}^{T} \boldsymbol{S}^{-1} \boldsymbol{M}\right)$. To obtain the optimum value of the upper bound of guaranteed cost, the term $\lambda_{\max }\left(\boldsymbol{M}^{T} \boldsymbol{S}^{-1} \boldsymbol{M}\right)$ in (23) is changed to

$$
\lambda_{\max }\left(\boldsymbol{M}^{T} \boldsymbol{S}^{-1} \boldsymbol{M}\right)<\lambda \Leftrightarrow \boldsymbol{M}^{T} \boldsymbol{S}^{-1} \boldsymbol{M}<\lambda \boldsymbol{I}
$$

which, in turn, implies the constraint (ii) in (14). Thus, the minimization of $(\varepsilon+\lambda)$ implies the minimization of the guaranteed cost in (23). The optimality of the solution of the optimization problem (14) follows from the convexity of the objective function and of the constraints. This completes the proof of Theorem 3.1.

Remark 3.1 It should be pointed out that the optimization problem given by (14) is an LMI eigenvalue problem $[52,53]$, which provides a procedure to design subo- ptimal guaranteed cost controller.

\section{Application to the Guaranteed Cost Control of Dynamical Processes Described by the Darboux Equation}

In this section, we shall demonstrate the application of our proposed method (Theorem 3.1) in robust guaranteed cost control of processes in the Darboux equation. It is known that some dynamical processes in gas absorption, water stream heating and air drying can be described by the Darboux equation $[3,7,8]$ :

$$
\frac{\partial^{2} s(x, t)}{\partial x \partial t}=a_{1} \frac{\partial s(x, t)}{\partial t}+a_{2} \frac{\partial s(x, t)}{\partial x}+a_{0} s(x, t)+b f(x, t)
$$

with the initial conditions

$$
s(x, 0)=p(x), \quad s(0, t)=q(t)
$$

where $s(x, t)$ is an unknown function at space $x \in\left[0, x_{f}\right]$ and time $t \in[0, \infty), a_{1}, a_{2}, a_{0}$ and $b$ are real constants and $f(x, t)$ is the input function.

Let

$$
r(x, t)=\frac{\partial s(x, t)}{\partial t}-a_{2} s(x, t)
$$

then (24) can be transformed into an equivalent system of first-order differential equation of the form:

$\left[\begin{array}{c}\frac{\partial r(x, t)}{\partial x} \\ \frac{\partial s(x, t)}{\partial t}\end{array}\right]=\left[\begin{array}{cc}a_{1} & a_{1} a_{2}+a_{0} \\ 1 & a_{2}\end{array}\right]\left[\begin{array}{l}r(x, t) \\ s(x, t)\end{array}\right]+\left[\begin{array}{l}b \\ 0\end{array}\right] f(x, t)$

It follows from (26) that

$r(0, t)=\left.\frac{\partial s(x, t)}{\partial t}\right|_{x=0}-a_{2} s(0, t)=\frac{\mathrm{d} q(t)}{d t}-a_{2} q(t)=z(t)$.

Taking

$$
\begin{aligned}
{\left[\begin{array}{ccc}
-\boldsymbol{S} & \overline{\boldsymbol{A}}_{1} & \overline{\boldsymbol{A}}_{2} \\
\overline{\boldsymbol{A}}_{1}^{T} & -\boldsymbol{Y} & \mathbf{0} \\
\overline{\boldsymbol{A}}_{2}^{T} & \mathbf{0} & -(\boldsymbol{S}-\boldsymbol{Y})
\end{array}\right] } & -\left[\begin{array}{ccccccc}
\boldsymbol{L} & \mathbf{0} & \mathbf{0} & \mathbf{0} & \mathbf{0} & \mathbf{0} \\
\mathbf{0} & \overline{\boldsymbol{M}}_{11} & \boldsymbol{S} \boldsymbol{Q}_{1}^{1 / 2} & \mathbf{0} & \boldsymbol{U}^{T} \boldsymbol{R}_{1}^{1 / 2} & \mathbf{0} \\
\mathbf{0} & \overline{\boldsymbol{M}}_{12} & \mathbf{0} & \boldsymbol{S} \boldsymbol{Q}_{2}^{1 / 2} & \mathbf{0} & \boldsymbol{U}^{T} \boldsymbol{R}_{2}^{1 / 2}
\end{array}\right] \\
& \times\left[\begin{array}{cccccc}
-\boldsymbol{I} & \mathbf{0} & \mathbf{0} & \mathbf{0} & \mathbf{0} & \mathbf{0} \\
\mathbf{0} & -\boldsymbol{I} & \mathbf{0} & \mathbf{0} & \mathbf{0} & \mathbf{0} \\
\mathbf{0} & \mathbf{0} & -\varepsilon \boldsymbol{I} & \mathbf{0} & \mathbf{0} & \mathbf{0} \\
\mathbf{0} & \mathbf{0} & \mathbf{0} & -\varepsilon \boldsymbol{I} & \mathbf{0} & \mathbf{0} \\
\mathbf{0} & \mathbf{0} & \mathbf{0} & \mathbf{0} & -\varepsilon \boldsymbol{I} & \mathbf{0} \\
\mathbf{0} & \mathbf{0} & \mathbf{0} & \mathbf{0} & \mathbf{0} & -\varepsilon \boldsymbol{I}
\end{array}\right]^{-1}\left[\begin{array}{ccc}
\boldsymbol{L}^{T} & \mathbf{0} & \mathbf{0} \\
\mathbf{0} & \overline{\boldsymbol{M}}_{11}^{T} & \overline{\boldsymbol{M}}_{12}^{T} \\
\mathbf{0} & \boldsymbol{Q}_{1}^{T / 2} \boldsymbol{S} & \mathbf{0} \\
\mathbf{0} & \mathbf{0} & \boldsymbol{Q}_{2}^{T / 2} \boldsymbol{S} \\
\mathbf{0} & \boldsymbol{R}_{1}^{T / 2} \boldsymbol{U} & \mathbf{0} \\
\mathbf{0} & \mathbf{0} & \boldsymbol{R}_{2}^{T / 2} \boldsymbol{U}
\end{array}\right]<\mathbf{0}
\end{aligned}
$$




$$
\begin{aligned}
& r(i, j)=r(i \Delta x, j \Delta t), \\
& s(i, j)=s(i \Delta x, j \Delta t), \\
& f(x, t)=u(i, j)
\end{aligned}
$$

and applying the forward difference quotients for both derivatives in (27), it is easy to verify that (27) can be expressed in the following form:

$$
\begin{aligned}
{\left[\begin{array}{l}
r(i, j) \\
s(i, j)
\end{array}\right] } & =\left[\begin{array}{cc}
\left(1+a_{1} \Delta x\right) & \left(a_{1} a_{2}+a_{0}\right) \Delta x \\
0 & 0
\end{array}\right]\left[\begin{array}{l}
r(i-1, j) \\
s(i-1, j)
\end{array}\right] \\
& +\left[\begin{array}{cc}
0 & 0 \\
\Delta t & \left(1+a_{2} \Delta t\right)
\end{array}\right]\left[\begin{array}{l}
r(i, j-1) \\
s(i, j-1)
\end{array}\right] \\
& +\left[\begin{array}{c}
b \Delta x \\
0
\end{array}\right] u(i-1, j)+\left[\begin{array}{l}
0 \\
0
\end{array}\right] u(i, j-1)
\end{aligned}
$$

with the initial conditions

$$
s(i, 0)=p(i \Delta x), \quad r(0, j)=z(j \Delta t) .
$$

By setting

$$
\boldsymbol{x}(i, j)=\left[\begin{array}{l}
r(i, j) \\
s(i, j)
\end{array}\right],
$$

(30) can be converted into the following FMSLSS model:

$$
\begin{aligned}
\boldsymbol{x}(i+1, & j+1)=\left[\begin{array}{cc}
0 & 0 \\
\Delta t & \left(1+a_{2} \Delta t\right)
\end{array}\right] \boldsymbol{x}(i+1, j) \\
& +\left[\begin{array}{cc}
\left(1+a_{1} \Delta x\right) & \left(a_{1} a_{2}+a_{0}\right) \Delta x \\
0 & 0
\end{array}\right] \boldsymbol{x}(i, j+1) \\
& +\left[\begin{array}{l}
0 \\
0
\end{array}\right] u(i+1, j)+\left[\begin{array}{c}
b \Delta x \\
0
\end{array}\right] u(i, j+1)
\end{aligned}
$$

with the initial conditions

$$
\boldsymbol{x}(i, 0)=\left[\begin{array}{c}
-a_{2} p(i \Delta x) \\
p(i \Delta x)
\end{array}\right], \quad \boldsymbol{x}(0, j)=\left[\begin{array}{l}
z(j \Delta t) \\
q(j \Delta t)
\end{array}\right]
$$

Now, consider the problem of suboptimal guaranteed cost control of a system represented by (33) with

$$
\begin{gathered}
a_{0}=\frac{1}{15}, \\
a_{1}=-1 \frac{3}{5}, \\
a_{2}=-\frac{1}{3}, \\
b=2, \\
\Delta x=0.5, \\
\Delta t=0.9
\end{gathered}
$$

and the initial conditions (34) satisfy (1g) and (1h) with

$$
p=q=2,
$$

$$
\boldsymbol{M}=\left[\begin{array}{cc}
0.01 & 0.05 \\
0.006 & 0.001
\end{array}\right] .
$$

It is also assumed that the above system is subjected to parameter uncertainties of the form (1c)-(1f) with

$$
\begin{gathered}
\boldsymbol{L}=\left[\begin{array}{l}
0 \\
1
\end{array}\right], \\
\boldsymbol{M}_{11}=\left[\begin{array}{ll}
0.0005 & 0
\end{array}\right], \\
\boldsymbol{M}_{12}=\left[\begin{array}{ll}
0 & 0.005
\end{array}\right], \\
\boldsymbol{M}_{21}=0, \\
\boldsymbol{M}_{22}=-0.007 .
\end{gathered}
$$

Associated with the uncertain system (33)-(37), the cost function is given by (2) with

$$
\begin{gathered}
\boldsymbol{Q}_{1}=\left[\begin{array}{cc}
0.09 & 0 \\
0 & 0.09
\end{array}\right], \\
\boldsymbol{Q}_{2}=\left[\begin{array}{cc}
0.9 & 0 \\
0 & 0.9
\end{array}\right], \\
\boldsymbol{R}_{1}=\boldsymbol{R}_{2}=0.0025 .
\end{gathered}
$$

Applying Lemma 2.1, it is easy to verify that the above system is unstable. We wish to construct a suitable guaranteed cost controller for this system, such that the corresponding cost bound is minimized. To this end, we apply our proposed method (Theorem 3.1) to find the suboptimal guaranteed cost controller. It is found using the LMI toolbox in Matlab [53] that the optimization problem (14) is feasible for the present example and the optimal solution is given by

$$
\begin{gathered}
\boldsymbol{S}=\left[\begin{array}{cc}
5.03810 & -4.53485 \\
-4.53485 & 7.41531
\end{array}\right], \\
\boldsymbol{Y}=\left[\begin{array}{cc}
1.22942 & 0.12961 \\
0.12961 & 1.19500
\end{array}\right], \\
\boldsymbol{U}=\left[\begin{array}{cc}
0.35283 & -1.31762
\end{array}\right], \\
\varepsilon=11.01117, \\
\lambda=0.00121 .
\end{gathered}
$$

By Theorem 3.1, the suboptimal guaranteed cost controller for this system is

$$
u(i, j)=\left[\begin{array}{ll}
-0.19999-0.29999
\end{array}\right] x(i, j),
$$

and the least upper bound of the corresponding closedloop cost function is

$$
J=0.02682 .
$$

\section{Conclusions}

In this paper, we have presented a method of designing a suboptimal guaranteed cost controller via static-state 
feedback for a class of 2-D discrete systems described by the FMSLSS model with norm bounded uncertainties. A suboptimal guaranteed cost controller is obtained through a convex optimization problem which can be solved by using Matlab LMI Toolbox [53]. Application of presented controller design method is demonstrated through processes described by a Darboux equation $[3,7,8]$. The presented method can also be applied for the robust guaranteed cost controller design for metal rolling control problem $[4,9,10]$.

\section{REFERENCES}

[1] N. K. Bose, "Applied Multidimensional System Theory," Van Nostrand Reinhold, New York, 1982.

[2] R. N. Bracewell, "Two-Dimensional Imaging," PrenticeHall, Englewood, 1995, pp. 505-537.

[3] C. Du, L. Xie and C. Zhang, " $H_{\infty}$ Control and Robust Stabilization of Two-Dimensional Systems in Roesser Models," Automatica, Vol. 37, No. 2, 2001, pp. 205-211. doi:10.1016/S0005-1098(00)00155-2

[4] S. Foda and P. Agathoklis, "Control of the Metal Rolling Process: A Multidimensional System Approach," Journal of the Franklin Institute, Vol. 329, No. 2, 1992, pp. 317 332. doi:10.1016/0016-0032(92)90037-H

[5] T. Kaczorek, "Two-Dimensional Linear Systems," SpringerVerlag, Berlin, 1985.

[6] W.-S. Lu and A. Antoniou, "Two-Dimensional Digital Filters,” Marcel Dekker, New York, 1992.

[7] W. Marszalek, "Two-Dimensional State-Space Discrete Models for Hyperbolic Partial Differential Equations," Applied Mathematical Modelling, Vol. 8, No. 1, 1984, pp. 11-14. doi:10.1016/0307-904X(84)90170-7

[8] J. S.-H. Tsai, J. S. Li and L.-S. Shieh, "Discretized Quadratic Optimal Control for Continuous-Time Two-Dimensional Systems," IEEE Transactions on Circuits and Systems I, Vol. 49, No. 1, 2002, pp. 116-125. doi:10.1109/81.974886

[9] M. Yamada, L. Xu and O. Saito, "2-D Model Following Servo System," Multidimensional Systems and Signal Processing, Vol. 10, No. 1, 1999, pp. 71-91. doi:10.1023/A:1008461019087

[10] R. Yang, L. Xie and C. Zhang, " $H_{2}$ and Mixed $H_{2} / H_{\infty}$ Control of Two-Dimensional Systems in Roesser Model," Automatica, Vol. 42, No. 9, 2006, pp. 1507-1514. doi:10.1016/j.automatica.2006.04.002

[11] R. P. Roesser, "A Discrete State-Space Model for Linear Image Processing," IEEE Transactions on Automatic Control, Vol. 20, No. 1, 1975, pp. 1-10. doi:10.1109/TAC.1975.1100844

[12] S. Attasi, "Modeling and Recursive Estimation for Double Indexed Sequences," In: R. K. Mehra and D. G. Lainiotis, Eds., System Identification: Advances and Case Studies, Academic, New York, 1976, pp. 289-348. doi:10.1016/S0076-5392(08)60875-9
[13] E. Fornasini and G. Marchesini, "State-Space Realization Theory of Two-Dimensional Filters," IEEE Transactions on Automatic Control, Vol. 21, No. 4, 1976, pp. 484-492. doi:10.1109/TAC.1976.1101305

[14] E. Fornasini and G. Marchesini, "Doubly Indexed Dynamical Systems: State-Space Models and Structural Properties," Theory of Computing Systems, Vol. 12, No. 1, 1978, pp. 59-72. doi:10.1007/BF01776566

[15] N. G. El-Agizi and M. M. Fahmy, "Two-Dimensional Digital Filters with No Overflow Oscillations," IEEE Transactions on Acoustics, Speech and Signal Processing, Vol. 27, No. 5, 1979, pp. 465-469. doi:10.1109/TASSP.1979.1163285

[16] J. H. Lodge and M. M. Fahmy, "Stability and Overflow Oscillations in 2-D State-Space Digital Filters," IEEE Transactions on Acoustics, Speech and Signal Processing, Vol. 29, No. 6, 1981, pp. 1161-1167. doi:10.1109/TASSP.1981.1163712

[17] B. D. O. Anderson, P. Agathoklis, E. I. Jury and M. Mansour, "Stability and the Matrix Lypunov Equation for Discrete 2-Dimensional Systems," IEEE Transactions on Circuits and Systems, Vol. 33, No. 3, 1986, pp. 261-267. doi:10.1109/TCS.1986.1085912

[18] P. Agathoklis, E. I. Jury and M. Mansour, "The Discrete-Time Strictly Bounded-Real Lemma and the Computation of Positive Definite Solutions to the 2-D Lyapunov Equation," IEEE Transactions on Circuits and Systems, Vol. 36, No. 6, 1989, pp. 830-837. doi: $10.1109 / 31.90402$

[19] D. Liu and A. N. Michel, "Stability Analysis of StateSpace Realizations for Two-Dimensional Filters with Overflow Nonlinearities," IEEE Transactions on Circuits and Systems I, Vol. 41, No. 2, 1994, pp. 127-137. doi: $10.1109 / 81.269049$

[20] H. Kar and V. Singh, "Stability Analysis of 2-D StateSpace Digital Filters Using Lyapunov Function: A Caution," IEEE Transactions on Signal Processing, Vol. 45, No. 10, 1997, pp. 2620-2621. doi:10.1109/78.640734

[21] H. Kar and V. Singh, "Stability Analysis of 2-D StateSpace Digital Filters with Overflow Nonlinearities," IEEE Transactions on Circuits and Systems I, Vol. 47, No. 4, 2000, pp. 598-601. doi:10.1109/81.841865

[22] H. Kar and V. Singh, "Stability Analysis of 1-D and 2-D Fixed-Point State-Space Digital Filters Using Any Combination of Overflow and Quantization Nonlinearities," IEEE Transactions on Signal Processing, Vol. 49, No. 5, 2001, pp. 1097-1105. doi:10.1109/78.917812

[23] T. Bose and D. A. Trautman, "Two's Complement Quantization in Two-Dimensional State-Space Digital Filters," IEEE Transactions on Signal Processing, Vol. 40, No. 10, 1992, pp. 2589-2592. doi:10.1109/78.157299

[24] T. Bose, "Stability of 2-D State-Space System with Overflow and Quantization," IEEE Transactions on Circuits and Systems II, Vol. 42, No. 6, 1995, pp. 432-434. doi:10.1109/82.392319

[25] Y. Su and A. Bhaya, "On the Bose-Trautman Condition for Stability of Two-Dimensional Linear Systems," IEEE 
Transactions on Signal Processing, Vol. 46, No. 7, 1998, pp. 2069-2070. doi:10.1109/78.700987

[26] A. Bhaya, E. Kaszkurewicz and Y. Su, "Stability of Asynchronous Two-Dimensional Fornasini-Marchesini Dynamical Systems," Linear Algebra and Its Applications, Vol. 332, 2001, pp. 257-263.

[27] H. Kar and V. Singh, "Stability of 2-D Systems Described by Fornasini-Marchesini First Model," IEEE Transactions on Signal Processing, Vol. 51, No. 6, 2003, pp. 1675-1676. doi:10.1109/TSP.2003.811237

[28] G.-D. Hu and M. Liu, "Simple Criteria for Stability of Two-Dimensional Linear Systems," IEEE Transactions on Signal Processing, Vol. 53, No. 12, 2005, pp. 47204723. doi:10.1109/TSP.2005.859265

[29] T. Zhou, "Stability and Stability Margin for a Two-Dimensional System," IEEE Transactions on Signal Processing, Vol. 54, No. 9, 2006, pp. 3483-3488. doi:10.1109/TSP.2006.879300

[30] T. Hinamoto, "2-D Lyapunov Equation and Filter Design Based on the Fornasini-Marchesini Second Model," IEEE Transactions on Circuits and Systems I, Vol. 40, No. 2, 1993, pp. 102-110. doi:10.1109/81.219824

[31] W.-S. Lu, "On a Lyapunov Approach to Stability Analysis of 2-D Digital Filters," IEEE Transactions on Circuits and Systems I, Vol. 41, No. 10, 1994, pp. 665-669. doi: $10.1109 / 81.329727$

[32] T. Ooba, "On Stability Analysis of 2-D Systems Based on 2-D Lyapunov Matrix Inequalities," IEEE Transactions on Circuits and Systems I, Vol. 47, No. 8, 2000, pp. 12631265. doi:10.1109/81.873883

[33] T. Hinamoto, "Stability of 2-D Discrete Systems Described by the Fornasini-Marchesini Second Model," IEEE Transactions on Circuits and Systems I, Vol. 44, No. 3, 1997, pp. 254-257. doi: $10.1109 / 81.557373$

[34] D. Liu, "Lyapunov Stability of Two-Dimensional Digital Filters with Overflow Nonlinearities," IEEE Transactions on Circuits and Systems I, Vol. 45, No. 5, 1998, pp. 574577. doi:10.1109/81.668870

[35] H. Kar and V. Singh, "An Improved Criterion for the Asymptotic Stability of 2-D Digital Filters Described by the Fornasini-Marchesini Second Model Using Saturation Arithmetic," IEEE Transactions on Circuits and Systems I, Vol. 46, No. 11, 1999, pp. 1412-1413. doi: $10.1109 / 81.802847$

[36] H. Kar and V. Singh, "Stability Analysis of 2-D Digital Filters Described by the Fornasini-Marchesini Second Model Using Overflow Nonlinearities," IEEE Transactions on Circuits and Systems I, Vol. 48, 2001, pp. 612-617.

[37] C. Du, L. Xie and Y. C. Soh, " $H_{\infty}$ Filtering of 2-D Discrete Systems," IEEE Transactions on Signal Processing, Vol. 48, No. 6, 2000, pp. 1760-1768. doi: $10.1109 / 78.845933$

[38] H. Kar and V. Singh, "Robust Stability of 2-D Discrete Systems Described by the Fornasini-Marchesini Second Model Employing Quantization/Overflow Nonlinearities,"
IEEE Transactions on Circuits and Systems II, Vol. 51, No. 11, 2004, pp. 598-602.

doi:10.1109/TCSII.2004.836880

[39] C. Du and L. Xie, "Stability Analysis and Stabilization of Uncertain Two-Dimensional Discrete Systems: An LMI Approach," IEEE Transactions on Circuits and Systems I, Vol. 46, No. 11, 1999, pp. 1371-1374. doi: $10.1109 / 81.802835$

[40] C. Du and L. Xie, "LMI Approach to Output Feedback Stabilization of 2-D Discrete Systems," International Journal of Control, Vol. 72, No. 2, 1999, pp. 97-106. doi:10.1080/002071799221262

[41] T. Bose, M. Q. Chen, K. S. Joo and G. F. Xu, "Stability of Two-Dimensional Discrete Systems with Periodic Coefficients," IEEE Transactions on Circuits and Systems II, Vol. 45, No. 7, 1998, pp. 839-847. doi: $10.1109 / 82.700930$

[42] X. Guan, C. Long and G. Duan, "Robust Optimal Guaranteed Cost Control for 2-D Discrete Systems," IET Control Theory \& Applications, Vol. 148, 2001, pp. 355-361.

[43] A. Dhawan and H. Kar, "LMI-Based Criterion for the Robust Guaranteed Cost Control of 2-D Systems Described by the Fornasini-Marchesini Second Model," Signal Processing, Vol. 87, No. 3, 2007, pp. 479-488. doi:10.1016/j.sigpro.2006.06.002

[44] A. Dhawan and H. Kar, "Optimal Guaranteed Cost Control of 2-D Discrete Uncertain Systems: An LMI Approach," Signal Processing, Vol. 87, No. 12, 2007, pp. 3075-3085. doi:10.1016/j.sigpro.2007.06.001

[45] S. S. L. Chang and T. K. C. Peng, "Adaptive Guaranteed Cost Control Systems with Uncertain Parameters," IEEE Transactions on Automatic Control, Vol. 17, No. 4, 1972, pp. 474-483. doi:10.1109/TAC.1972.1100037

[46] I. R. Petersen and D. C. Mcfarlane, "Optimal Guaranteed Cost Control and Filtering for Uncertain Linear Systems," IEEE Transactions on Automatic Control, Vol. 39, No. 9, 1994, pp. 1971-1977. doi:10.1109/9.317138

[47] I. R Petersen, D. C. Mcfarlane and M. A. Rotea, "Optimal Guaranteed Cost Control of Discrete-Time Uncertain Linear Systems," International Journal of Robust and Nonlinear Control, Vol. 8, No. 8, 1998, pp. 649-657. doi:10.1002/(SICI)1099-1239(19980715)8:8<649::AID-R $\mathrm{NC} 334>3.0 . \mathrm{CO} ; 2-6$

[48] M. S. Mahmoud and L. Xie, "Guaranteed Cost Control of Uncertain Discrete Systems With Delays," International Journal of Control, Vol. 73, No. 2, 2000, pp. 105-114. doi:10.1080/002071700219812

[49] H. Mukaidani, "An LMI Approach to Guaranteed Cost Control for Uncertain Delay Systems," IEEE Transactions on Circuits and Systems I, Vol. 50, No. 6, 2003, pp. 795-800. doi:10.1109/TCSI.2003.812620

[50] X. Nian and J. Feng, "Guaranteed Cost Control of a Linear Uncertain System with Multiple Time-Varying Delays: An LMI Approach," IET Control Theory \& Applications, Vol. 150, 2003, pp. 17-22.

[51] L. Xie and Y. C. Soh, "Guaranteed Cost-Control of Uncertain Discrete-Time Systems," Control Theory and Ad- 
vanced Technology, Vol. 10, 1995, pp. 1235-1251.

[52] S. Boyd, L. El Ghaoui, E. Feron and V. Balakrishnan, "Linear Matrix Inequalities in System and Control Theory," SIAM, Philadelphia, 1994. doi:10.1137/1.9781611970777

[53] P. Gahinet, A. Nemirovski, A. J. Laub and M. Chilali, "LMI Control Toolbox-for Use with Matlab," The MATH Works Inc., Natick, 1995.

[54] V. Balakrishnan and E. Feron, "Linear Matrix Inequalities in Control Theory and Applications," International Journal of Robust Nonlinear Control, Special Issue, 1996, pp. 896-1099.

[55] L. El Ghaoui and S. I. Niculescu, "Advances in Linear Matrix Inequality Methods in Control, Advances in Design and Control," SIAM, Philadelphia, 2000.

[56] T. Iwasaki, "LMI and Control," Shokodo, Japan, 1997.

[57] Y. Nesterov and A. Nemirovskii, "Interior-Point Polynomial Algorithms in Convex Programing," SIAM, Philadelphia, 1994.

[58] L. Vandenberghe and V. Balakrishnan, "Algorithms and Software for LMI Problems in Control," IEEE Control Systems, Vol. 17, No. 5, 1997, pp. 89-95. doi: $10.1109 / 37.621480$

[59] S. Xu, J. Lam, Z. Lin and K. Galkowski, "Positive Real Control for Uncertain Two-Dimensional Systems," IEEE Transactions on Circuits and Systems I, Vol. 49, No. 11, 2002, pp. 1659-1666. doi:10.1109/TCSI.2002.804531

[60] P. P. Khargonekar, I. R. Petersen and K. Zhou, "Robust Stabilization of Uncertain Linear Systems: Quadratic Stability and $H_{\infty}$ Control Theory," IEEE Transactions on Automatic Control, Vol. 35, 1991, pp. 356-361.

[61] L. Xie, M. Fu and C. E. De Souza, " $H_{\infty}$ Control and Quadratic Stabilization of Systems with Parameter Uncertainty via Output Feedback," IEEE Transactions on Automatic Control, Vol. 37, No. 8, 1992, pp. 1253-1256. doi:10.1109/9.151120

[62] F. Yang and Y. S. Hung, "Robust Mixed $H_{2} / H_{\infty}$ Filtering with Regional Pole Assignment for Uncertain Discrete-Time Systems," IEEE Transactions on Circuits and Systems I, Vol. 49, No. 8, 2002, pp. 1236-1241. doi:10.1109/TCSI.2002.801267

[63] M. S. Mahmoud, "Robust Control and Filtering for TimeDelay Systems,” Marcel-Dekker, New York, 2000. 